\title{
FACTORIZATION OF CERTAIN MAPS UP TO HOMOTOPY
}

\author{
GEORGE KOZLOWSKI ${ }^{1}$
}

If $f: X \rightarrow Y$ is a map of a space $X$ into a space $Y$, we say that $f$ is a local connection in dimension $n$, provided that for every point $y \in Y$ and every neighborhood $N$ of $y$ there is a neighborhood $V \subset N$ of $y$ such that for $0 \leqq k \leqq n-1$ any map $g: S^{k} \rightarrow f^{-1} V$ extends to a map $g^{\prime}: B^{k+1} \rightarrow f^{-1} N$ and for any map $g: S^{n} \rightarrow f^{-1} V$ the map $f g: S^{n} \rightarrow V$ extends to a map $h: B^{n+1} \rightarrow N$. Using star-refinements of open covers and a standard approximation technique we establish the following theorem (a slightly weaker form of which has been announced by Price [2]).

Theorem 1. Let $Y$ be a metric space, and let $f: X \rightarrow Y$ be a local connection in dimension $n$ wrth dense image. Let $L$ be a subcomplex of a finite simplicial complex $K$ such that $\operatorname{dim}(K-L) \leqq n$, and let $g: L \rightarrow X$ and $h: K \rightarrow Y$ be maps such that $h \mid L=$ fg. Then there is a map $g^{\prime}: K \rightarrow X$ such that $g^{\prime} \mid L=g$ and $f g^{\prime}$ is homotopic to $h$ relative to $L$. If $d$ is any metric for $Y$ and $\epsilon>0$, the map $g^{\prime}$ and the homotopy $H$ may be chosen so that for all points $p \in K$ the diameter (with respect to $d$ ) of $H(p \times I)$ is $<\epsilon$.

This implies that $f$ is an $n$-equivalence; i.e. $f$ maps the set of pathcomponents of $X$ bijectively to the set of path-components of $Y$, and that for every $x \in X, f \sharp: \pi_{k}(X, x) \subset \pi_{k}(Y, f(x))$ is an isomorphism for $1 \leqq k \leqq n-1$ and an epimorphism for $k=n$. Since $f \mid f^{-1} W$ is also a local connection in dimension $n$ for every open set $W \subset Y$, it follows that $Y$ is $L C^{n}$. Using these facts and the lemmas for the proof of Theorem 1 we obtain sharper forms of known results:

Theorem 2 (cF. Smale [3]). Let $X$ be a paracompact $\mathrm{LC}^{n}$ space, let $Y$ be a metric space, and let $f: X \rightarrow Y$ be a closed map of $X$ onto $Y$ such that $f^{-1}(y)$ is $\mathrm{LC}^{n-1}$ and $(n-1)$-connected for every $y \in Y$. Then $Y$ is $\mathrm{LC}^{n}$, and $f$ is an n-equivalence.

Theorem 3 (CF. KWUn [1]). Let $M$ be a manifold, and let $G$ be an upper semicontinuous decomposition of $M$ into cellular sets. If the

\footnotetext{
Received by the editors February 14, 1968.

${ }^{1}$ The author was supported by an NSF Graduate Fellowship and by NSF GP. 6462 .

These results are contained in the author's Ph.D. Thesis (University of Michigan), written under the direction of Professor Morton Brow'n.
} 
decomposition space $M / G$ is finite dimensional, it is a homotopy manifold.

1. All complexes will be finite simplicial complexes, and $(K, L)$ will be called an $n$-pair provided that $L$ is a subcomplex of $K$ and $\operatorname{dim}(K-L) \leqq n$. The $q$-skeleton of $K$ will be denoted $K^{q}$. If $\mathcal{u}$ is a collection of open sets in $Y$, then a map $h: K \rightarrow Y$ (a homotopy $H: K \times I \rightarrow Y$ ) will be said to map $K$ (resp. $K \times I$ ) into $\mathcal{u}$ provided that for every (closed) simplex $\alpha$ of $K$ there is $U \in \mathcal{U}$ with $h(\alpha)$ $\subset U$ (resp. $H(\alpha \times I) \subset U)$. Associated with $\mathcal{u}$ is the collection $\mathfrak{u}^{*}$ $=\left\{U^{*} \mid U \in \mathcal{U}\right\}$, where $U^{*}=U\left\{U^{\prime} \in \mathcal{U} \mid U \cap U^{\prime} \neq \varnothing\right\}$. A map $f: X \rightarrow Y$ will be said to be a strong local connection in dimension $n$, if for every point $y \in Y$ and every neighborhood $N$ of $y$ there is a neighborhood $V \subset N$ of $y$ such that for $0 \leqq k \leqq n$ any map $g: S^{k} \rightarrow f^{-1} V$ extends to a $\operatorname{map} g^{\prime}: B^{k+1} \rightarrow f^{-1} N$.

Let $\mathcal{U}$ and $\mathcal{V}$ be open covers of a space $Y$ such that $\vartheta$ refines $\mathcal{u}$, and let $f: X \rightarrow Y$ be a map. For every nonnegative integer $n$ we define assertions $E(\mathcal{V}, \mathfrak{u} ; n), H(\mathcal{V}, \mathcal{u} ; n)$, and $H(\mathcal{V}, \mathcal{u} ; f ; n)$ as follows:

$E(\mathcal{V}, \mathcal{u} ; n)$. If $(K, L)$ is any $n$-pair and $g: L \rightarrow X, h: K \rightarrow Y$ are any maps such that $h$ extends $f g$ and maps $\operatorname{cl}(K-L)$ into the collection $\{V \in V \mid f(X) \cap V \neq \varnothing\}$, then there is an extension $g^{\prime}: K \rightarrow X$ of $g$ such that for every simplex $\alpha$ of $\operatorname{cl}(K-L)$ there is $U \in \mathcal{u}$ with $f g^{\prime}(\alpha) \cup h(\alpha) \subset U$.

$H(\mathcal{U}, \mathcal{u} ; n)$. If $(K, L)$ is any $n$-pair and $g: L \rightarrow X, g^{\prime}: K \rightarrow X$, $g^{\prime \prime}: K \rightarrow X$ are any maps such that $g=g^{\prime}\left|L=g^{\prime \prime}\right| L$ and for every simplex $\alpha$ of $K$ there is $V \in \mathcal{V}$ with $g^{\prime}(\alpha) \cup g^{\prime \prime}(\alpha) \subset f^{-1} V$, then there is a homotopy $G: g^{\prime} \simeq g^{\prime \prime}$ relative to $L$ which maps $K \times I$ into $f^{-1} u$ $=\left\{f^{-1} U \mid U \in \mathcal{U}\right\}$.

$H(\mathcal{V}, \mathcal{u} ; f ; n)$. If $(K, L)$ is any $n$-pair and $g: L \rightarrow X, g^{\prime}: K \rightarrow X$, $g^{\prime \prime}: K \rightarrow X$ are any maps such that $g=g^{\prime}\left|L=g^{\prime \prime}\right| L$ and for every simplex $\alpha$ of $K$ there is $V \in \mathcal{V}$ with $g^{\prime}(\alpha) \cup g^{\prime \prime}(\alpha) \subset f^{-1} V$, then there is a homotopy $H: f g^{\prime} \simeq f g^{\prime \prime}$ relative to $L$ which maps $K \times I$ into $\mathcal{u}$.

Lemma 1. Let $Y$ be paracompact, and let $f: X \rightarrow Y$ be a strong local connection in dimension $n$. Then for any open cover $\mathcal{U}$ of $Y$ there is an open cover $\mathcal{v}$ of $Y$ refining $\mathcal{u}$ such that both $E(\mathcal{V}, \mathcal{u} ; n+1)$ and $H(\mathcal{v}, \mathcal{u} ; n)$ hold.

Proof. For $n=-1$ there are no conditions on the $\operatorname{map} f$, and both assertions hold for $v=\mathcal{~}$. Assume that the lemma is true for $n<k$, and let $f: X \rightarrow Y$ be a strong local connection in dimension $k$. If $u$ is an open cover of $Y$, let $W$ be an open cover such that for each $W \in W$ there is $U \in \mathcal{U}$ such that $W^{*} \subset U$ and any map $S^{k} \rightarrow f^{-1}\left(W^{*}\right)$ extends to a map $B^{k+1} \rightarrow f^{-1} U$. 
Let $v$ be an open cover refining $W$ such that both $E(v, w ; k)$ and $H(\mathcal{V}, \mathfrak{W} ; k-1)$ hold. Then both $E(\mathcal{v}, \mathfrak{u} ; k+1)$ and $H(\mathcal{v}, \mathfrak{u} ; k)$ hold. In fact if $K, L, g, h$ are as in the first assertion, there is an extension $g^{\prime \prime}: L \cup K^{k} \rightarrow X$ such that for every $k$-simplex $\beta$ of $\operatorname{cl}(K-L)$ there is $W(\beta) \in W$ with $f g^{\prime \prime}(\beta) \cup h(\beta) \subset W(\beta)$. Let $\alpha$ be a $(k+1)$-simplex of $K-L$, and let $W \in W$ be such that $h(\alpha) \subset W$. If $\beta<\alpha$, then $W \cap W(\beta)$ $\neq \varnothing$. It follows that $f g^{\prime \prime}(\partial \alpha) \subset W^{*}$; hence there is $U \in \mathcal{U}$ such that $W^{*} \subset U$, and there is an extension $\alpha \rightarrow f^{-1} U$ of $g^{\prime \prime} \mid \partial \alpha: \partial \alpha \rightarrow f^{-1}\left(W^{*}\right)$. Combining such extensions gives the desired map $g^{\prime}: K \rightarrow X$; thus $E(\mathcal{V}, \mathcal{u} ; k+1)$ holds. On the other hand if $K, L, g, g^{\prime}, g^{\prime \prime}$ are as in the second assertion, let $G^{\prime}:\left(L \cup K^{k-1}\right) \times I \rightarrow X$ be a homotopy as in $H(\mathcal{V}, W ; k-1)$, and extend $G^{\prime}$ to $G^{\prime \prime}: K \times\{0,1\} \cup\left(L \cup K^{k-1}\right) \times I \rightarrow X$ by $G^{\prime \prime}(p, 0)=g^{\prime}(p)$ and $G^{\prime \prime}(p, 1)=g^{\prime \prime}(p)$ for all points $p \in K$. For any $k$-simplex $\alpha$ of $K$ there is $W \in W$ such that $G^{\prime \prime}(\alpha \times\{0,1\}) \subset f^{-1} W$; thus $G^{\prime \prime}(\partial(\alpha \times I)) \subset f^{-1}\left(W^{*}\right)$. Then $G^{\prime \prime}$ extends to a homotopy $G: K \times I \rightarrow X$ which maps $K \times I$ into $u$, which proves that $H(\mathcal{\vartheta}, u ; k)$ holds.

Lemma 2. Let $Y$ be paracompact, and let $f: X \rightarrow Y$ be a local connection in dimension $n$. Then for any open cover $u$ of $Y$ there is an open cover $\mathcal{v}$ refining $\mathfrak{U}$ such that $H(\mathcal{U}, \mathcal{u} ; f ; n)$ holds.

Proof. If $n=-1$, there is nothing to prove. Assume that the lemma is true for $n<k$, and let $f: X \rightarrow Y$ be a local connection in dimension $k$. Then $f$ is a strong local connection in dimension $k-1$. If $\mathcal{u}$ is an open cover of $Y$, let $W$ be an open cover such that for each $W \in W$ there is $U \in \mathcal{u}$ such that $W^{*} \subset U$ and for any map $h: S^{k} \rightarrow f^{-1}\left(W^{*}\right)$ the map $f h$ extends to a map $B^{k+1} \rightarrow U$. By Lemma 1 there is an open cover $V$ of $Y$ refining w such that $H(v, W ; k-1)$ holds. To see that $H(\mathcal{v}, u ; f ; k)$ holds consider $K, L, g, g^{\prime}$, and $g^{\prime \prime}$ as in the assertion, and let $G:\left(L \cup K^{k-1}\right) \times I \rightarrow X$ be a homotopy as in $H(\mathcal{V}, W ; k-1)$. Extend $G$ to a map $G^{\prime}: K \times\{0,1\} \cup\left(L \cup K^{k-1}\right)$ $X I \rightarrow X$ by $G^{\prime}(p, 0)=g^{\prime}(p)$ and $G^{\prime}(p, 1)=g^{\prime \prime}(p)$, and observe that for every $k$-simplex $\alpha$ of $K-L$ there is $W \in W$ with $f G^{\prime}(\partial(\alpha \times I)) \subset W^{*}$. It follows that $f G^{\prime}$ extends to a homotopy $H$ which maps $K \times I$ into $u$.

2. Proof of Theorem 1. Let $Y$ have metric $d$. Using Lemmas 1 and 2 choose a sequence $\left\{V_{r} \mid 0 \leqq r<\infty\right\}$ of open covers of $Y$ such that mesh $v_{r}<\epsilon / 4(r+1), \quad V_{r+1}^{*}$ refines $V_{r}, E\left(V_{r+1}, V_{r} ; n\right)$ holds, and $H\left(V_{r+1}^{*}, v_{r} ; f ; n\right)$ holds. (For a given $\epsilon$ such a sequence provides the extension and the homotopy in all cases.)

If $K, L, g$, and $h$ are as in Theorem 1 , choose a sequence $\left\{K_{r} \mid 1 \leqq r<\infty\right\}$ of subdivisions of $K$ such that $K_{r+1}$ is a subdivision 
of $K_{r}$ and $h$ maps $K_{r}$ into $\mho_{r+1}$. Using the fact that $E\left(v_{r+1}, \vartheta_{r} ; n\right)$ holds choose extensions $g_{r}: K_{r} \rightarrow X$ of $g(1 \leqq r<\infty)$ such that for every $\alpha$ in $K_{r}$ there is $V \in v_{r}$ with $f g_{r}(\alpha) \cup h(\alpha) \subset V$. Since mesh $v_{r}<\epsilon / 4(r+1)$, $d\left(f g_{r}(p), h(p)\right)<\epsilon / 4(r+1)$ for all points $p \in K$.

Set $g^{\prime}=g_{1}$, and construct $H$ by "filling in" between $g_{r}$ and $g_{r+1}$ as follows. Let $\alpha$ be a simplex of $K_{r}$, and let $f g_{r}(\alpha) \cup h(\alpha) \subset V$ for some $V \in V_{r}$. Consider $\alpha$ as a subcomplex of $K_{r+1}$, and observe that for every simplex $\beta$ of $\alpha$, there is $V^{\prime} \in \mathcal{V}_{r}$ such that $f g_{r+1}(\beta) \cup h(\beta) \subset V^{\prime}$; hence $f g_{r+1}(\alpha) \cup f g_{r}(\alpha) \subset V^{*}$. Since $H\left(V_{r}^{*}, v_{r-1} ; f ; n\right)$ holds, there is a homotopy $H_{r}: f g_{r+1} \sim f g_{r}$ relative to $L$, which may be considered as a map $H_{r}: K \times[1 /(r+1), 1 / r] \rightarrow Y$, such that the diameter of $H_{r}(\alpha \times[1 /(r+1), 1 / r])$ is $<\epsilon / 4 r$ for every simplex $\alpha$ of $K_{r}$. This implies that $d\left(H_{r}(p, t), h(p)\right)<\epsilon / 2 r$ for all $(p, t) \in K \times[1 /(r+1), 1 / r]$. Define $H: K \times I \rightarrow Y$ by $H(p, t)=H_{r}(p, t)$ for $1 /(r+1) \leqq t \leqq 1 / r$ and by $H(p, 0)=h(p)$. It is easy to check that $H$ is a map and is in fact an $\epsilon$-homotopy relative to $L$. This completes the proof.

3. Proof of Theorem 2. Since $f$ is a closed map and $f^{-1}(y)$ is $(n-1)$ connected for every $y \in Y$, to show that $f$ is a local connection in dimension $n$ it suffices to show that for every open neighborhood $U$ of $f^{-1}(y)$ there is an open neighborhood $V \subset U$ of $f^{-1}(y)$ such that for $0 \leqq k \leqq n$ any map $S^{k} \rightarrow V$ is homotopic in $U$ to a map $S^{k} \rightarrow f^{-1}(y)$. Let $A$ be the inverse set under $f$ of a point of $Y$. Since for any closed $\mathrm{LC}^{n-1}$ subset $A$ of an $\mathrm{LC}^{n}$ space $X$, the inclusion map $i: A \subset X$ is a local connection in dimension $n$, and since $X$ is paracompact, Lemma 1 applies to $i: A \subset X$ and $1: X \subset X$. If $U$ is an open neighborhood of $A$, let $\mathcal{u}$ be the cover of $X$ consisting of $U$ and $X-A$, let $W$ be an open cover of $X$ refining $\mathfrak{u}$ such that $H(w, \mathfrak{u} ; n)$ holds for $1: X \subset X$, and let $U$ be an open cover refining $W$ such that $E(V, W ; n)$ holds for $i: A \subset X$.

Set $V=U\left\{V^{\prime} \in U \mid V^{\prime} \cap A \neq \varnothing\right\}$. For $0 \leqq k \leqq n$ triangulate $S^{k}$ in some way as a complex $K$, and observe that for any map $h: K \rightarrow V$ there is a subdivision $K^{\prime}$ of $K$ such that $h$ maps $K^{\prime}$ into $\left\{V^{\prime} \in \mathcal{V} \mid V^{\prime} \cap A \neq \varnothing\right\}$. It follows that there is a map $g: K^{\prime} \rightarrow A$ such that for each $\alpha$ in $K^{\prime}$ there is $W \in W$ with $g(\alpha) \cup h(\alpha) \subset W$. Since $H(W, u ; n)$ holds for $1: X \subset X$, there is a homotopy $H: h \simeq g$ : $K^{\prime} \times I \rightarrow X$ such that $H$ maps $K^{\prime} \times I$ into $\mathcal{~}$. Since $g\left(K^{\prime}\right) \subset A$, $H\left(K^{\prime} \times I\right) \subset U$. This completes the proof.

4. Proof of Theorem 3. We recall that $M$ is an $n$-manifold, if it is a separable metric space each point of which has an open neighborhood homeomorphic to $R^{n}$ and that a subset $A$ of $M$ is cellular, if $A=\bigcap_{j-1}^{\infty} Q_{j}^{n}$, where $Q_{j}^{n}$ is a closed $n$-cell $(1 \leqq j<\infty)$ and int $Q_{j}^{n} \supset Q_{j+1}^{n}$. 
If $G$ is an upper semicontinuous decomposition of $M$ into cellular subsets, then it is well known that $M / G$ is a separable metric space and that the projection $P: M \rightarrow M / G$ is a closed map. It follows directly from the definition of cellularity that $P$ is a strong local connection in dimension $k$ for all $k$, and therefore that $M / G$ is $\mathrm{LC}^{\infty}$. In order to prove that for every point $x \in M / G$ and every neighborhood $N$ of $x$ there are (connected) open neighborhoods $V, U$ of $x$ such that $V \subset U \subset N$ and for all $k$ the image of $\pi_{k}(V-x)$ in $\pi_{k}(U-x)$ (under the homomorphism induced by the inclusion $V-x \subset U-x$ ) is isomorphic to $\pi_{k}\left(S^{n-1}\right)$ we could duplicate the arguments of [1] using the fact that $P \mid P^{-1}(W)$ is a local connection in all dimensions for every open set $W$ of $M / G$ wherever Smale's theorem is used. We shall omit these details.

\section{REFERENCES}

1. K. W. Kwun, $A$ fundamental theorem on decompositions of the sphere into points and tame arcs, Proc. Amer. Math. Soc. 12 (1961), 47-50.

2. T. M. Price, On decompositions and homotopy groups, Notices Amer. Math. Soc. 14 (1967), 274.

3. S. Smale, $A$ Vietoris mapping theorem for homotopy, Proc. Amer. Math. Soc. 8 (1957), 604-610.

UNIVERSITY OF Michigan 